\title{
Auditory Illusions as Evidence for a Role of the Syllable in Adult Developmental Dyslexics
}

\author{
Beatrice de Gelder $*$ $\dagger$ ANd JeAn VROOMEN* \\ *Tilburg University, The Netherlands; and †Universite Libre de Bruxelles, Belgium
}

\begin{abstract}
This study investigated whether adult developmental dyslexics differ from normal controls in early stages of spoken language processing that in turn might be related to specific reading difficulties. Subjects were required to detect prespecified targets under dichotic presentation of auditory nonword pairs. The stimuli were made such that segment migrations were possible. The potential contribution of phonetic features, as well as that of phonemes and syllables, was investigated. The results showed that dyslexics had specifically more syllable-based migrations. This result is discussed in the context of well-documented metaphonological deficits and memory problems of poor readers. (c) 1996 Academic Press, Inc.
\end{abstract}

\section{INTRODUCTION}

There is considerable agreement that the aspect of language processing that is critically involved in reading-acquisition difficulties concerns phonological representations (for recent overviews, see Bertelson, 1987; Liberman, Shankweiler, \& Liberman, 1989; Rack, Snowling, \& Olson, 1992). The notion of a deficit in phonological processing covers a wide spectrum of possibilities. Among those that have been extensively explored are phonological memory disorders. Equally well charted is the impairment in metaphonological abilities of young poor readers. In contrast, the possibility of anomalies in speech processes is one that has received very little attention and for which very little evidence is available. The present research pursues this latter avenue and addresses the issue of speech perception anomalies in poor readers. The concern is not with poor reading as such, but with a possible insight

This study was presented previously at the XIth Cognitive Neuropsychology workshop in Bressanone, January 1991, and at the Tenet IV meeting in Montreal, May 1993. Thanks to M. van Zon and T. Popelier for assistance with testing the subjects. Research reported in the present paper was supported partially by a grant from the Belgian Ministere de l'Education de la Communauté Française ("Action de recherche concertée,' Language processing in different modalities: Comparative approaches). Address correspondence and reprint requests to Beatrice de Gelder, Tilburg University, Department of Psychology, P.O. Box 90153, 5000 LE Tilburg, The Netherlands. Fax: +31-13662370. E-mail: degelder@kub.nl. 
into poor reading acquisition. This means that evidence from the detailed study of how poor readers deal with written language and insights to be gained from looking into their strategies is not of primary importance here.

As we will argue, it might be fruitful to investigate the stages of processing of speech sounds that are possibly independent of and come before lexical access and word recognition. The task used for this purpose here is one of examining speech misperceptions that occur as a consequence of dichotic fusions of speech segments, whether features, phonemes, or syllables. A specific aspect of the present study is its focus on adult developmental dyslexics. Generally, it is agreed that some deficits characteristic of young poor readers may disappear over time and might thus be a matter of a developmental lag rather than a core deficit (Campbell \& Butterworth, 1985; Stanovich, Nathan, \& Zolman, 1988). Since studies of adult developmental dyslexics point to aspects of reading-acquisition failure that persist over time, they might be helpful to clarify the delay versus deficit controversy and show which aspects of language processing remain anomalous.

\section{Phonological Awareness, Phonological Memory, and the Relation to Speech Perception}

In comparison with memory and metaphonological ability, speech perception is the least explored of the aspects of poor reading skills. Yet there is some evidence related to it, direct as well as indirect. The indirect evidence concerning the involvement of speech processing is mostly inconclusive or negative. It is evidence that comes from studies that have tried to establish either metaphonological skills or poor phonological memory as the principle causal factor. We briefly review these findings and claims before reviewing direct and positive, but so far limited, evidence for speech-processing problems.

The first and best explored concomitant of poor reading concerns poor metaphonological skills. Poor readers show impairments in the ability to manipulate subsyllabic segments in the typical tasks used to assess phonological awareness (Bradley \& Bryant, 1983). Several studies (e.g., Lieberman, Meskill, Chatillon, \& Schupack, 1985; Pratt \& Brady, 1988) have found evidence for impaired phoneme awareness in poor readers. In line with those findings, Pennington, van Orden, Smith, \& Green (1990) conclude in their study of adult dyslexics that phoneme awareness stands out as the best candidate for a primary deficit of dyslexia and note that this is most clearly so in the subject group of dyslexics with a clear hereditary lineage.

While it would bring us too far to enter into the debate on metaphonological skills, we must briefly consider the issue of the relation of these skills with reading and with on-line speech processing abilities. None of the above studies has made an explicit claim about the relation between poor metaphonological skills and reading ability. We have argued elsewhere that the notion 
of metaphonological skills is descriptive rather than explanatory (de Gelder, 1990) and so have others (Cossu, Rossini, \& Marshall, 1993). One consistent position has been to assign metaphonological abilities to a separate domain insulated from modular speech-processing abilities (Bertelson \& de Gelder, $1989,1991)$. The drawback of this view is that it leads to a dualist picture of unrelated phonological and metaphonological abilities. However, it is a picture that so far does seem to fit the results obtained with adults who are poor readers but still have good metaphonological skills (de Gelder \& Vroomen, 1991) and it also goes together with metaphonologically unable children who are good readers (Cossu et al., 1993).

We turn next to a brief discussion of memory problems of poor readers to the extent that these touch on issues of speech-processing deficits. The situation here is similar to that with metaphonological studies just reviewed. Studies of memory performance of poor readers have not made any direct claims about explanations of poor memory performance that would lay outside the domain of memory skill per se. As theories of memory become more sophisticated, their potential for explaining memory impairments by appealing to impaired components of the memory system seems to increase. A major factor of poor memory on young readers seems to be the relatively less efficient use of the phonological loop, a subsystem of memory in Baddeley's theory (see Hulme \& Mackenzie, 1992, for a recent overview). In this context strong claims are made about a causal role of memory impairments for reading-acquisition difficulties. In a recent study of dyslexics' memory performance, we discussed whether impaired memory performance might reveal something about speech-processing deficits (de Gelder \& Vroomen, 1995) and concluded against a direct inference from memory performance to speech processing. Using a serial-recall task it was found that the memory impairment of adult developmental dyslexics was clear only for the middle serial positions. If poor memory has its origin in poor speech processing (for example, less discriminable speech representations), one would expect to find a reduced recency effect, like the one observed when acoustically similar items have to be recalled. Instead, data from poor readers show normal recency. Some other studies have looked at speech perception to see whether it could be ruled out as an alternative explanation of poor memory. Baddeley and researchers (e.g., Gathercole \& Baddeley, 1990) examined language-impaired subjects that were poor readers and found typical memory impairments in the absence of phoneme discrimination problems. The task used required a similarity judgement between a pair of words or non-words differing on the first phoneme. Gathercole and Baddeley (1990), as well as Pennington et al. (1990), reach negative conclusions about the possibility that poor speech perception skills explain reading disorders, yet the tasks used focus on speech processing only indirectly. In the study of Pennington et al. (1990) the conclusion is based upon what the authors call a phoneme perception task. In fact it is a task which requires subjects to repeat a word. 
In the Gathercole and Baddeley study, subjects are asked to make a similarity judgement (even so, the language-impaired group is poorer than the controls). Neither of these tasks relate to speech perception per se.

\section{A Speech-Processing Deficit: Which Focus on Processing and Which Locus of Impairment?}

The few available studies that have looked directly at speech processing in the reading impaired do provide suggestive evidence for a more focused and specific phonological processing deficit than the metaphonological skills and memory problems discussed above. The literature contains few suggestions as to the possible loci of speech-processing deficits. Some of these have effectively come up in research on poor readers, but others are implicit in recent speech processing studies. Poor readers are less able to decode speech in noise (Brady, Shankweiler, \& Mann, 1983). One suggestion for a locus of these problems is that speech processing in poor readers might be characterized by poor feature distinction or extraction and ensuing poor phoneme identification. In studies using an auditory categorical perception tasks, differences between good and poor readers were observed (Godfrey, SyrdalLasky, Millay, \& Knox, 1981; Werker \& Tees, 1987). Our own research confirms this picture (de Gelder \& Vroomen, 1989). Interestingly, we have observed an impairment with heard as well as with lip-read speech, which would suggest that the locus of the deficit might well be in the phonetic processor rather than being a matter of low-level acoustic factors (de Gelder \& Vroomen, 1988; de Gelder \& Vroomen, 1995).

It thus seems that the general issue of whether poor readers have speech perception problems is at present not settled. There is direct and indirect evidence which leads to different conclusions. In the present study, a more fine-grained analysis was undertaken to investigate whether one of the other segments, i.e., features, phonemes, or syllables, might be critically implicated in phonological anomalies. The paradigm used in this experiment was designed to elicit speech misperceptions following dichotic presentation of stimulus pairs. The methodology of illusory conjunctions adopted here was derived from the research on work on visual perception and the role played by object parts in the preperceptive stages of object recognition by Treisman and collaborators (e.g., Treisman, 1986). The paradigm has been transposed to the study of written language perception since the work by Prinzmetal (1981) and more recently with the study of letter migrations (Kolinsky, 1992; Mozer, 1983; McClelland \& Mozer, 1986). Antecedents of the paradigm in the domain of spoken language processing include the study of fusions by Cutting (1976). Along these lines it was observed that dichotic presentation of a pair of spoken words may lead to the mistaken perception of a target (Kolinsky, Morais, \& Cluytens, 1995). An example from a related domain is the McGurk illusion (McGurk \& MacDonald, 1976), where an illusory 
perception of a /da/ is generated by combining an auditory /ba/ and a visual /ga/. The dichotic fusion technique has been used to study anomalous lateralization of language functions in developmental language-disordered or reading-disordered populations (Bryden, 1988; Kershner and Morton, 1990). The basic logic of this paradigm can be stated as follows. When subjects are presented simultaneously with conflicting information, the perceptual system generates a percept that is a combination of the processing properties of the system and of the input. What results is a perceptual illusion that may not correspond to the two inputs. On this account, perceptual illusions offer a window on the architecture of the processing system and allow us to study possible speech-processing problems. The logic of this paradigm may therefore fit our current guesses about possible speech-processing problems in developmental dyslexics.

The goal of the present experiment was to investigate whether poor readers would be different from normals in the stages of speech processing that precede lexical access. Therefore, the task was designed with pseudowords rather than real words. This choice was also motivated by a framework for speech perception anomalies in developmental dyslexics, i.e., the notion of a prelexical deficit which in due course is covered up by semantic processes involved in word recognition. This notion is convergent with Treisman's ideas suggesting that word knowledge might explain the very limited occurrence of conjunction errors in real life. First of all, we were apprehensive that, in a task using word targets, lexical bias might overrule any abnormality that might be present in poor readers at the prelexical level. If a phonological processing deficit does indeed exist and is related to impaired reading acquisition, it must clearly be a subtle one, as apparently it goes unnoticed in normal perception and production of meaningful speech of the reading impaired. Dichotic presentation allowed us to look at the circumstances of fusions. The actual hypothesis this experiment was designed to address is whether dyslexics would be impaired in speech processing and if a specific locus could be targeted at a level of segments like features, phonemes, or syllables. Such a finding would then allow us to address the theoretical question on the difficulties of this population in acquiring an alphabetic orthography that represents phonemes.

In this study we looked at a population of adult developmental dyslexics who suffered from specific reading deficits with a well-documented history of reading-acquisition difficulties. Following accepted criteria (e.g., Seymour, 1986), the subjects can be qualified as developmental dyslexics. The task they were asked to perform was one of target detection following dichotic presentation of nonword pairs. The stimuli were designed in such a way as to generate target illusions that would follow from recombination of specific parts of each item in the pairs. The goal of the experiment was to find out whether the dyslexic group suffered more than the normal subjects from speech misperceptions and whether there was a bias in these mispercep- 
tions that might be a consequence of the kind of segment (feature, phoneme, or syllable) that made the critical difference between the pairs and target.

\section{METHOD}

Subjects. Two groups of subjects were studied. One consisted of 15 adult dyslexics ( 9 male, 6 female, mean age $=32: 1$ years, range between 17 and 51 years). The control group consisted of 14 adult subjects (5 male, 9 female) of comparable age and socioeconomic background. The subjects of the dyslexic group all had a long history of reading difficulties that had become manifest at the time they were first taught reading in school. The dyslexic subjects were given a reading test (Brus 1-minute test) and a pseudo-word reading test as used in previous work (de Gelder \& Vroomen, 1991). The dyslexic subjects read 53.9 words per minute (range from 18 to 77 ) and 29.7 pseudowords (range from 17 to 51). For comparison, normal adults read about 100 words and 74 pseudo-words per minute (see de Gelder \& Vroomen, 1991). All subjects were right handed and reported normal hearing. None of these subjects were known to suffer from other deficits, linguistic or other. All subjects were paid for their participation.

Stimuli. The materials consisted of eight CVCV pseudowords which served as targets (see Appendix A). The initial consonant of the targets was always a plosive. The targets had to be detected under dichotic presentation. Our main interest was to investigate migrations at the feature, phoneme, and syllable level. Therefore, in the experimental conditions, items were presented which differed from the target in the initial feature (place or voicing), phoneme, or syllable. These items were paired with words which did have the "missing" feature, phoneme, or syllable in the right position. As an example, we will describe construction for the target word bieno. In the experimental feature condition, the item pieno was presented to one ear, and it was paired with the word dатти presented to other ear. Fusion of the word pieno with the voicing feature of the $d$ from dammu results in bieno. Similarly, in the phoneme condition, the word tieno was paired with bатmu, and in the syllable condition tanno was paired with biemu. In the control conditions, the items did not have the critical migration information. Thus, instead of dатти in which the voicing feature of $d$ was crucial, we presented the unvoiced таттии; in the phoneme condition катти replaced ватти, and in the syllable condition реeти replaced biети. The word pairings described so far formed the "no" conditions in which the target word itself (bieno) was actually not present. In order to perform signaldetection analyses, we also presented the target word (in this example bieno) requiring a "yes" response, and paired it with items used in the "no" condition (as described above: bieno was paired with dатти, ьатти, and віети for the experimental conditions, and with tатти, катти, and реети for the control conditions). Orthogonal combination resulted in 3 (feature, phoneme, syllable $) \times 2$ (experimental, control $) \times 2$ (target present/target absent $)=12$ conditions. Half of the word pairs were presented with the target word on the left ear, and for the other half, the target word was presented on the right ear.

Preparation of stimuli and design. The items were spoken by a male speaker of Dutch. The items were first recorded on a tape recorder (Revox P99), and then digitized at $16 \mathrm{kHz}$. Then, the onset of the plosive was determined visually using a speech waveform editor. The onset was defined as the first sustained excursion above the noise level, followed by clear periodicity. The word pairs were then low-pass-filtered at $7.8 \mathrm{kHz}$ and played back on a DAT recorder with the onset of the plosives synchronized. The peak intensities of the input channels were matched on the VU meters. For each of the eight targets, there were 20 repetitions such that each condition is based on 160 measurements per subject. Each target word was presented in 10 consecutive blocks of 24 items. Within each block, each of the 12 conditions appeared twice in random order with the restrictions that no more than three target-present or targetabsent trials appeared in a row, and that no more than three presentations of a critical word were presented to the same ear in a row. The order of the target words was varied across subjects. 
Procedure. Subjects were tested individually in a sound-proof booth, listening over headphones (AKG K100) at approximately $65 \mathrm{~dB}$ SPL. Items were presented via a digital audio tape recorder (Phillips DAT 850). Subjects were told that they would hear two nonwords simultaneously, one to each ear. Their task was to identify a prespecified target nonword in either the left or the right ear. To prevent intrusions, target words were written on a card in front of the subject. They had to push a response button with the preferred index. The word pairs were preceded by a beep which acted as a warning signal. The intertrial interval was 4 sec. After 120 trials there was a small pause, and after $2 \mathrm{hr}$ there was a 30-min rest. Testing lasted about $4 \mathrm{hr}$. After each pause, there were 6 warm-up trials. Prior to testing, subjects were given 120 practice trials with a target and word pairs that were not used later in experimental testing.

\section{RESULTS}

Individual numbers of hits and false alarms (FA) were determined for each group (dyslexics and controls), trial type (experimental versus control), and segment (feature, phoneme, and syllable). Hits and FA rates were transformed into d-prime scores (Green \& Swets, 1966). The mean d-primes are shown in Table 1. As expected, experimental trials had lower d-primes than control trials. This difference is attributed to migrations of the relevant segment. Dyslexics had overall lower d-primes than controls and more migrations at the syllable level.

An Analysis of Variance (ANOVA) was performed on the d-prime scores with group (dyslexics, controls) as between-subjects factor, and with segment (feature, phoneme, syllable) and condition (experimental, control) as within-subjects factors. The main effect of group was significant because dyslexics had lower d-prime scores than the controls $[F(1,27)=10.50, p$ $<$.003]. There was also a main effect of segment, mainly because features had lower d-primes than phonemes and syllables $[F(2,54)=47.76, p<$ $.001]$. The difference between experimental and control pairs was highly significant $[F(1,27)=103.38, p<.001]$, indicating that d-primes were lower in the experimental conditions. Of interest were significant interactions between group and condition $[F(1,27)=8.38, p<.007]$, between segment and condition $[F(2,54)=9.84, p<.001]$, and the second-order interaction between group, segment, and condition $[F(2,54)=4.01, p<.024]$. To investigate the latter interaction, separate ANOVAs were performed for each

\section{TABLE 1}

Mean d-prime Scores for Each Group and for Each Segment in the Control and Experimental Trials

\begin{tabular}{|c|c|c|c|c|c|c|}
\hline & \multicolumn{2}{|c|}{ Feature } & \multicolumn{2}{|c|}{ Phoneme } & \multicolumn{2}{|c|}{ Syllable } \\
\hline & Control & Exp & Control & Exp & Control & Exp \\
\hline Dyslexics & 1.64 & 1.30 & 2.04 & 1.92 & 2.33 & 1.62 \\
\hline Controls & 2.21 & 1.80 & 2.60 & 2.60 & 2.71 & 2.47 \\
\hline
\end{tabular}


segment. For features, there was a significant difference between dyslexics and control subjects $[F(1,27)=7.54, p<.011]$, and between experimental and control trials $[F(1,27)=32.42, p<.001]$, but the interaction between group and condition was not significant $(F<1)$. For phonemes, there was a difference between dyslexics and controls $[F(1,27)=8.11, p<.008]$, but neither was the difference between experimental and control pairs significant, nor did the interaction reach significance. Finally, with syllables dyslexics had lower d-primes than controls $[F(1,27)=12.33, p<.002]$, experimental trials had lower d-primes than control trials $[F(1,27)=76.31$, $p<.001]$, and the interaction between group and condition was highly significant $[F(1,27)=18.87, p<.001]$. Separate $t$ tests on the d-primes of the syllabic control and experimental trials showed that d-primes of the control trials of dyslexics were slightly lower than those from the control group $[t(27)=1.89, p=.06]$, but this difference was much larger for the experimental trials $[t(27)=4.83, p<.001]$. It thus shows that dyslexics had many more syllable migrations than control subjects.

\section{DISCUSSION}

The goal of this study was to investigate whether anomalies exist in the early stages of spoken language processing of adult developmental dyslexics. The illusory perception paradigm was used because of its potential for investigating the contrastive role of different linguistic segments between two groups of native speakers. Our hypothesis was that the dyslexics would be different from normal controls in that they might show a different pattern of illusions. The results show that such a difference in auditory illusions is indeed observed between the two groups. The critical difference is not found in the feature or phoneme, but in the syllable condition. The feature condition does generate migration illusions for normals and dyslexics alike; in the phoneme condition there is no migration at all, and only in the syllable condition are the scores such that one can conclude that there are more migrations in the dyslexic group. Dyslexics were-compared to normal controls-thus more likely to detect an illusory target such as bieno when presented with the dichotic pair tanno-biemmu.

One very general comment is that this finding is in line with the notion, well supported by the bulk of research on developmental dyslexia of the last two decades, that phonological processing is the critical locus of abnormal performance of poor readers. Beyond this very general issue, this result highlights the possible relevance of one so far neglected domain of phonological processes, i.e., speech processing. As the present task did not involve any critical reading skills, the poor result of the dyslexic group reflects a spokenlanguage-processing deficit. Given the adult age of the subjects, it also appears to be a persistent deficit. Most critically, the observed syllable-based illusion of the dyslexics suggests that the syllable plays a special role in 
speech processing in this group. In what follows, we discuss these issues and examine how the result relates to current insights about poor reading acquisition.

\section{Acoustic Factors, Reading Ability, and Memory}

The speech signal is a very complex one and it would be an understatement to say that the information relative to perceiving a syllable, consonant, or feature is not contained in a transparent fashion. This is not to say that the acoustic realization of the various concepts used in phonology is the same. Coarticulation, the major source of opacity of the speech signal, is more intense within syllables than across syllables (Liberman \& Mattingly, 1985). In the preparation of the material the necessary precautions were taken in order to avoid a confounding of phonological and acoustic factors, but it remains possible that acoustic factors have selective effects. For that reason it is hazardous to make comparisons over segments and to claim that, for instance, syllables migrate more or less than features or phonemes. However, once we look at contrasts within a given segment between two different groups, inferences are more secure because both groups hear exactly the same acoustic material. In this way, factors other than those related to the speech processing architecture can be ruled out as confounding the result.

If that point is granted, one might object that it is the difference in reading ability that is responsible for the result and not a difference in speechprocessing architecture. One interpretation of this objection would consist of arguing that the results reflect an orthographic bias which is not present or is not as efficient in dyslexics. It must be remembered, however, that the targets were nonwords, and that they were presented auditorily. This procedure was chosen to minimize the impact of orthographic skill. Still, orthographic knowledge might have an effect in some more subtle ways. One might be that normal readers have a better or more readily available letter knowledge. What we do not rule out, at this stage, is that there are influences from orthographic knowledge that affect the stages in between early parsing or speech segmentation and conscious report on segmentation tasks, but this is at present a rather unexplored domain.

Another concern relates to the memory requirements in the dichotic task. Although memory load is minimal, the issue of a possible effect can be raised since in general adult dyslexics do have a somewhat impaired phonological memory. In an earlier study investigating a very similar population of adult dyslexics, we concluded that the impaired memory was limited to a matter of reduced span. Critical aspects of the serial-recall performance of poor readers, whether young or adult, tended to be very much like those of normal subjects, whether the input was auditory or required lipreading. Most importantly, the recency and the suffix effects resemble those of normal subjects, i.e., clear recency for speech lists (in the heard as well as in the lip-read 
modality) and also clear suffix effects (de Gelder \& Vroomen, in press). As we noted, good memory does not preclude the possibility of poor speech representations in the dyslexic group: it only suggests that speech processing and memory may be relatively independent and that there may be a dissociation between them, as was reported for acquired phonological dyslexics (Martin \& Breedin, 1992).

\section{Reading Ability and the Development of Speech Processing}

We now turn to the main issue of possible explanations of the selective involvement of the syllable segment in poor readers and speculate on a possible developmental explanation for this effect. To push this issue, it is worth looking into a recent discussion of phonological alexia patients by Patterson and Marcel (1992) who sketch a symmetry between the analysis they propose and reading development. Patterson and Marcel note that the issue of acquired reading impairment is not that remote from the question on the units of processing. This observation would be valid a fortiori for reading-acquisition disorders, although, as we noted in the Introduction, this is not a concern that has occupied the forefront of reading development research. Exceptions are Marcel (1980) and Studdert-Kennedy (1981), who have argued that the observation of speech errors at the phoneme level points to the existence of phonemes as prelexical units. The patients discussed by Patterson and Marcel do resemble our phonological dyslexics in some respects, such as poor reading of nonwords, and are significantly different in others, since they have very reduced digit span and, most importantly, impaired metaphonological skills. As part of an explanation of this pattern they sketch a picture of reduced intentional access to subsyllabic representations. Such reduced access would be a major handicap for doing phonological assembly as required in reading nonwords. As they point out, this picture rests on a view of normal language development as bringing about access to more analytical or more fine-grained levels of phonology. This is the accepted view on metaphonological skills we referred to in the Introduction. To give a more detailed example, the notion is that in the course of development children go from an ability to manipulate syllables to being able to segment into onset/rine and finally into phonemes. The notion of a retreat to less analytical units in acquired phonological reading disorders could thus be the mirror image of a blocked access to more analytical units in the developmental case. But there is also a possible asymmetry. Since, before their trauma, phonological alexics were normal readers, there is no reason to take seriously an impairment in their speech-processing stages. Their problem may thus be one of access to more fine-grained representations, while the problem of the developmental dyslexics may be one of poor availability in the sense of blocked access. As Patterson and Marcel suggest, the difficulty of phonological alex- 
ics with manipulating low-level or subsyllabic manipulations may stem from an impaired access. The presupposition of this explanation is that these patients have intact subsyllabic representations. This presupposition needs to be investigated in the case of developmental dyslexics. But the ambiguity between impaired access and reduced availability needs to be unpacked in the case of developmental dyslexics. In a study comparing explicit phonemic segmentation and implicit comparison we found that adult phonological dyslexics were good at the former task while being poor at the latter, more implicit task that might better reflect phonological processing (de Gelder \& Vroomen, 1991). This observation suggests a possible dissociation between segmentation skills and phonological assembly required in reading.

What looms beyond this issue of the locus of a syllable effect in speech processing is the more fundamental question of how to characterize the phonological knowledge of the prereaders and the ability to acquire skills for grapheme/phoneme conversions critical for normal alphabetic reading skills. At present very little is known about the child's phonological knowledge at the age where reading tuition starts. There is increasing evidence that phonological development takes place during the first years and may go on well beyond that, partly in relation with lexical development and lexical organization. Against this background, anomalous phonological development can be a matter of a relative failure to develop fine-grained representations, whether or not these are under the control of language-specific processes such as those at stake in becoming a native listener (Werker, 1989) or those in acquiring the phonotactics of the native language (Friederici \& Wessels, 1993; Jusczyk, 1986). The notion of a developmental disorder in the acquisition of subsyllabic processing routines cannot be substantiated at present. It appears extremely difficult to bring such a development in focus, as it may well occur around the age when, in Western societies, formal tuition in alphabetic skills starts. Studies of normally functioning adult developmental dyslexics should be helpful to direct attention to underlying aspects of readingacquisition difficulties that may be a matter of a proper spoken language deficit rather than developmental delay in learning to read.

\section{APPENDIX A}

Target word: BIENO

Target absent

Target present

\begin{tabular}{|c|c|c|c|c|}
\hline & Experimental & Control & Experimental & Control \\
\hline Feature: & pieno-dammu & pieno-tammu & bieno-dammu & bieno-tammu \\
\hline Phoneme: & tieno-bammu & tieno-kammu & bieno-bammu & bieno-kammu \\
\hline Syllable: & tanno-biemu & tanno-peemu & bieno-biemu & bieno-peemu \\
\hline
\end{tabular}




\section{REFERENCES}

Bertelson, P. 1987. The onset of literacy. In P. Bertelson (Ed.), The onset of literacy: Cognitive processes in reading acquisition. Cambridge, MA: MIT Press.

Bertelson, P., \& de Gelder, B. 1989. Learning about reading from illiterates. In A. M. Galaburda (Ed.), From reading to neurons. Cambridge, MA: MIT Press.

Bertelson, P., \& de Gelder, B. 1991. The emergence of phonological awareness. In I. Mattingly \& M. Studdert-Kennedy (Eds.), The motor theory of speech perception. Hillsdale, NJ: Erlbaum.

Braddy, S., Shankweiler, D., \& Mann, V. A. 1983. Speech perception and memory coding in relation to reading ability. Journal of Experimental Child Psychology, 35, 345-367.

Bradley, L., \& Bryant, P. E. 1983. Categorizing sounds and learning to read: A causal connection. Nature, 301, 419-421.

Campbell, R., \& Butterworth, B. 1985. Phonological dyslexia and dysgraphia in a highly literate subject: A developmental case with associated deficits in phonemic processing and awareness. Quarterly Journal of Experimental Psychology, 37A, 435-475.

Cossu, G., Rossini, F., \& Marshall, J. C. 1993. When reading is acquired but phonemic awareness is not: A study of literacy in Down's syndrome. Cognition, 46, 129-138.

Cutting, J. E. 1976. Auditory and linguistic processes in speech perception: Inferences from six fusions in dichotic listening. Psychological Review, 83, 114-140.

de Gelder, B. 1990. Phonological awareness, misidentification and multiple identities. In C. Feagin \& P. Muhlhauser (Eds.), Development and diversity: Linguistic variation across time and space. Arlington, Texas Publications.

de Gelder, B., \& Vroomen, J. 1988. Do developmental dyslexics have less robust speech categories? Paper presented at the 6th Cognitive Neuropsychology Workshop, Bressanone.

de Gelder, B., \& Vroomen, J. 1991. Phonological Deficits: beneath the surface of readingacquisition problems. Psychological Research, 53, 88-97.

de Gelder, B., \& Vroomen, J. 1995. Phonological memory deficits in young and adult dyslexics. In B. de Gelder and J. Morais (Eds.), Language and literacy. Hillsdale, NJ: Erlbaum.

Friederici, A. D., \& Wessels, M. I. 1993. Phonotactic knowledge of word boundaries and its use in infant speech perception. Perception and Psychophysics, 54, 287-295.

Gathercole, S. E., \& Baddeley, A. D. 1990. Phonological memory deficits in language disordered children: Is there a causal connection? Journal of Memory and Language, 29, 336360 .

Godfrey, J. J., Syrdal-Lasky, A. K., Millay, K. K., \& Knox, C. M. 1981. Performance of dyslexic children on speech perception tests. Journal of Experimental Child Psychology, 32, 401-424.

Green, D. M., \& Swets, J. A. 1966. Signal detection theory and psychophysics. New York: Krieger.

Hulme, C., \& Mackenzie, S. 1992. Working memory and severe learning difficulties. Hillsdale, NJ: Erlbaum.

Hurford, D. P., \& Sanders, R. E. 1990. Assessment and remediation of a phonemic discrimination deficit in reading disabled second and fourth graders. Journal of Experimental Child Psychology, 50, 396-415.

Jusczyk, P. W. 1986. Towards a model for the development of speech perception. In J. Perkell \& D. H. Klatt (Eds.), Invariance and variability in speech processes. Hillsdale, NJ: Erlbaum.

Kershner, J. R., \& Morton, L. L. 1990. Directed attention dichotic listening in reading disabled children: A test of four models of maladaptive lateralization. Neuropsychologia, 28, 181198.

Kolinsky, R. 1992. Conjunction errors as a tool for the study of perceptual processing. In J. 
Alegria, D. Holender, J. Morais, \& M. Radeau (Eds.), Analytic approaches to human cognition. Amsterdam: Elsevier.

Kolinsky, R., Morais, J., \& Cluytens, M. 1995. Intermediate representations in spoken word recognition: Evidence from word illusions. Journal of Memory and Language, 34, 1940.

Liberman, A. M., \& Mattingly, I. G. 1985. The motor theory of speech perception revised. Cognition, 21, 1-36.

Liberman, I. Y., Shankweiler, D., \& Liberman, A. M. 1989. The alphabetic principle and learning to read. In D. Shankweiler \& I. Y. Liberman (Eds.), Phonology and reading disability: Solving the reading puzzle. IARLD Research Monograph Series. Ann Arbor: University of Michigan Press.

Lieberman, P., Meskill, R. H., Chatillon, M., \& Schupack, H. 1985. Phonetic speech perception deficits in dyslexia. Journal of Speech and Hearing Research, 28, 480-486.

Marcel, A. J. 1980. Surface dyslexia and beginning reading: A revised hypothesis of the pronunciation of print and its impairments. In M. Coltheart, K. Patterson, \& J. C. Marshall (Eds.), Deep dyslexia. London: Routledge.

Martin, R. C., \& Breedin, S. D. 1992. Dissociations between speech perception and phonological short-term memory deficits. Cognitive Neuropsychology, 9, 509-534.

McClelland, J. L., \& Mozer, M. C. 1986. Perceptual interactions in two-word displays: Familiarity and similarity effects. Journal of Experimental Psychology: Human Perception and Performance, 12, 18-35.

McGurk, H., \& MacDonald, J. 1976. Hearing lips and seeing voices. Nature, 264, 746-748.

Mozer, M. C. 1983. Letter migration in word perception. Journal of Experimental Psychology: Human Perception and Performance, 9, 531-546.

Patterson, K., \& Marcel, A. 1992. Phonological ALEXIA or PHONOLOGICAL alexia. In J. Alegria, D. Holender, J. Morais, and M. Radeau (Eds.), Analytic approaches to human cognition. Amsterdam: Elsevier.

Pennington, B. F., van Orden, G. C., Smith, S. D., \& Green, P. A. 1990. Phonological processing skills and deficits in adult dyslexics. Child Development, 61, 1753-1778.

Pratt, A. C., \& Brady, S. 1988. Relation of phonological awareness to reading disability in children and adults. Journal of Educational Psychology, 80, 319-323.

Prinzmetal, W. 1981. Principles of feature integration in visual perception. Perception and Psychophysics, 30, 330-340.

Rack, J. P., Snowling, M. J., \& Olson, R. K. 1992. The nonword reading deficit in developmental dyslexia: A review. Reading Research Quarterly, 27, 28-53.

Seymour, P. H. K. 1986. Cognitive analysis of dyslexia. London: Routledge and Kegan Paul.

Stanovich, K. E., Nathan, R. G., \& Zolman, J. E. 1988. The developmental lag hypothesis in reading: Longitudinal and matched reading-level comparisons. Child Development, 59, $71-86$.

Studdert-Kennedy, M. 1981. The emergence of phonetic structure. Cognition, 10, 301-306. Treisman, A. 1986. Features and objects in visual processing. Scientific American, 255, 114b125.

Werker, J. F. 1989. Becoming a native listener. American Scientist, 77, 54-59.

Werker, J. F., \& Tees, R. C. 1987. Speech perception in severely disabled and average reading children. Canadian Journal of Psychology, 41, 48-61. 